\title{
Erratum to: Motor coordination: when two have to act as one
}

\author{
Daniel A. Braun • Pedro A. Ortega • \\ Daniel M. Wolpert
}

Published online: 30 June 2011

(c) Springer-Verlag 2011

\section{Erratum to: Exp Brain Res (2011) 211:631-641}

DOI 10.1007/s00221-011-2642-y

In the original publication of this article, in Fig. 4a, b, the green and blue colours have been wrongly assigned and displayed. The correct figure is placed here along with its caption.

The online version of the original article can be found under doi:10.1007/s00221-011-2642-y.

D. A. Braun $(\bowtie) \cdot$ P. A. Ortega · D. M. Wolpert

Department of Engineering, Computational and Biological,

Learning Laboratory, University of Cambridge, Cambridge, UK

e-mail: dab54@cam.ac.uk 
Fig. 4 a, b Joint entropy and mutual information between the distribution of positions of Player 1 and Player 2. All trajectories were discretized into 10 equidistant points, and the positions of these points were categorized (see "Methods" for details). The average over coordinated trajectories is shown in blue, and the average over miscoordinated trajectories is shown in green. During the movement, the joint entropy is increased for coordinated solutions in the coordination, stag hunt and battle of sexes game. The mutual information between the two players is always elevated at the end of the movement for all games compared with miscoordinated trials. c, d Dependence of coordination probability on initial positions of Player 1 and Player 2. For initial positions close to the corners of the workspace, the coordination probability at the end of the trial is increased. The error bars in d are obtained through bootstrapping
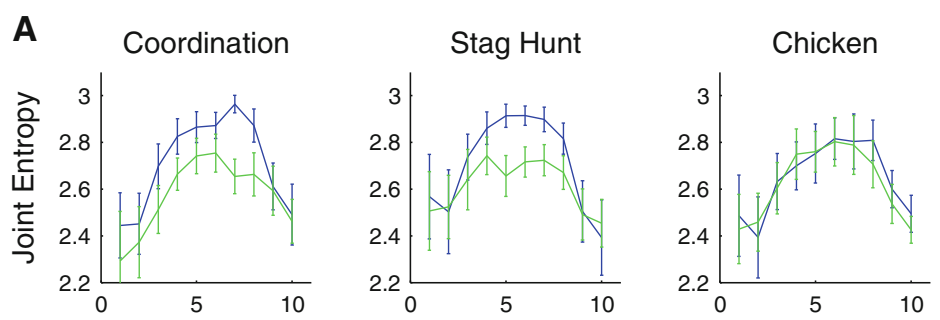

Battle of Sexes
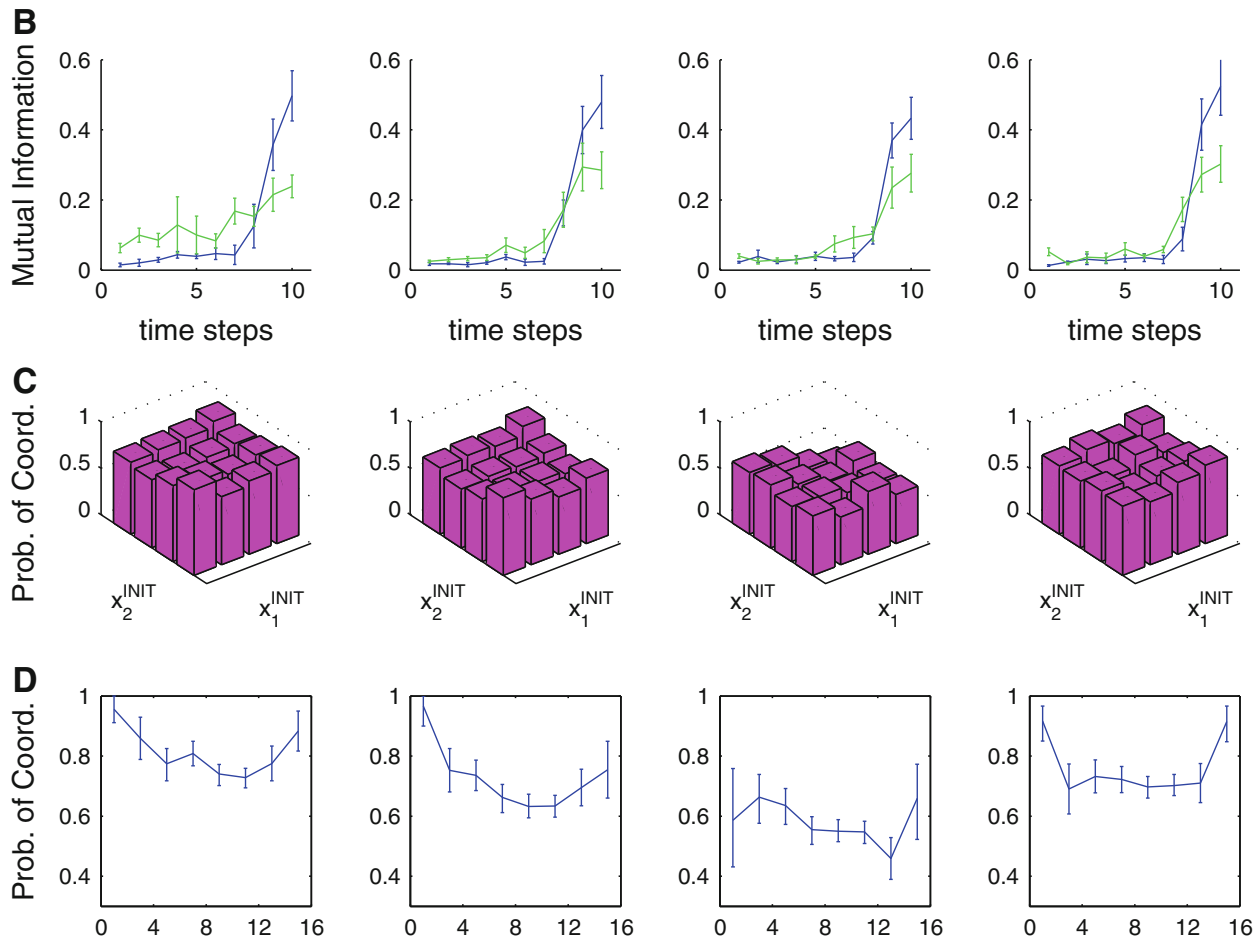

Init. dist. to Nash (cm) Init. dist. to Nash (cm) Init. dist. to Nash (cm) Init. dist. to Nash (cm) 